\title{
ON THE 2-DECOMPOSITION NUMBERS OF $\operatorname{Sp}(4, q)$
}

\author{
DONALD L. WHITE
}

Let $G$ be the finite symplectic group $\operatorname{Sp}(4, q)$, where $q$ is a power of an odd prime $p$. In this note we provide the 2-decomposition numbers of $G$ (with some ambiguity in the principal block); that is, the multiplicities of the characteristic 2 irreducible Brauer characters in the reduction modulo 2 of the ordinary irreducible characters. It is hoped that explicit results such as these and related results of Shamash [3] and Hiss [2] will yield some insight into the general problem of determining the modular representations of finite groups of Lie type in characteristics other than the defining characteristic of the group.

The ordinary character table of $G$ was computed by Srinivasan [4], and we use the notation of that paper for the characters. The ordinary characters fall naturally into indexed families corresponding to families of conjugacy classes. Similarly, the 2-blocks of $G$ fall into families corresponding to families of classes of semisimple elements of odd order in the dual group $G^{*}$ of $G$. There is a one-to-one correspondence between the individual blocks and classes of these elements such that the decomposition matrix of each block is exactly the same as that of the principal block of the dual of the centralizer in $G^{*}$ of elements in the corresponding class.

The decomposition numbers are shown in the tables below. The details of the distribution of characters into blocks appear in [5] and are omitted here for simplicity. Instead, we indicate from which families the characters in a given block are taken, and give the number of characters in the block from each family.

The notation in the tables is as follows:

$$
q-1=2^{n} m, \quad q+1=2^{n^{\prime}} m^{\prime}
$$

where $m$ and $m^{\prime}$ are odd integers,

$$
d=n+n^{\prime}
$$

is the 2-part of $q^{2}-1, \varepsilon= \pm 1$ is such that $q \equiv \varepsilon(\bmod 4)$, and $\alpha=1+\varepsilon$. The entry labelled $x$ in Table 4 is 1 if $q=3$, but in general is known only to be an integer,

$$
0 \leq x \leq(q-1) / 2 \text {. }
$$

The blocks of types $b_{1}, b_{2}, b_{3}, b_{4}$, and $b_{5}$ are exactly those with abelian defect group (and, consistent with Brauer's height conjecture, exactly those

Received by the editors August 13, 1987.

1980 Mathematics Subject Classification (1985 Revision). Primary 20C30; Secondary $20 \mathrm{C20}$.

Research partially supported by NSF Grant \#DMS-8512904. 
in which every character is of height zero). In each case either the defect group is cyclic or the direct product of a cyclic group and the center (of order 2 ), or the inertial index of the block is 1 . It follows that each of these blocks contains exactly one Brauer character and all decomposition numbers are 1. (See Feit [1, Theorem V.9.13 and Chapter VII].)

The decomposition numbers of the principal block $b_{0}$ and blocks of types $b_{67}, b_{89}, b_{1}$ and $b_{I I I}$ are more difficult to obtain. The primary technique used in the proof is the restriction of characters to a subgroup of odd order, namely a Sylow $p$-subgroup $U$ of $G$. It is shown that if

$$
\left\{\varphi_{j}: j=1, \ldots, l(b)\right\}
$$

is the set of Brauer characters in a block $b$ of one of these types, then there is a set $\left\{\psi_{k}: k=1, \ldots, r\right\}$ of ordinary irreducible characters of $U$, with $r=l(b)$ or $l(b)-1$, such that the multiplicity of $\psi_{k}$ in the restriction of $\varphi_{j}$ to $U$ is $\delta_{j k}$. It follows that if $\chi \in b$ is an ordinary irreducible character, then the first $r$ decomposition numbers relative to $\chi$ are simply the multiplicities of the $\psi_{k}$ in the restriction of $\chi$ to $U$. Thus we obtain all but at most one column of the decomposition matrix for each block. Using various properties of the decomposition numbers we are able to determine the matrix completely except in the case of the principal block, where some ambiguity remains in the final column. All Brauer characters are known except those corresponding to columns 2 and 3 in the principal block, although it is known that these are conjugate under a diagonal outer automorphism.

Details of the proof may be found in [5] and will appear elsewhere.

$$
\begin{aligned}
& b_{1}\left(\frac{q^{2}-1}{8} \text { blocks }\right) \text { : } \\
& \begin{array}{c|c|c|c}
\text { degrees } & \text { chars. } & & \begin{array}{c}
\text { no. of } \\
\text { chars. }
\end{array} \\
\hline\left(q^{2}-1\right)^{2} & \chi_{1} & 1 & 2
\end{array} \\
& b_{2}\left(\frac{(m-1)\left(m^{\prime}-1\right)}{4} \text { blocks }\right): \\
& \begin{array}{c|c|c|c}
\text { degrees } & \text { chars. } & & \begin{array}{c}
\text { no. of } \\
\text { chars. }
\end{array} \\
\hline q^{4}-1 & \chi_{2} & 1 & 2^{d}
\end{array} \\
& b_{3}\left(\frac{(m-1)(m-3)}{8} \text { blocks }\right): \\
& \begin{array}{l|c|c|c}
\text { degrees } & \text { chars. } & & \begin{array}{c}
\text { no. of } \\
\text { chars. }
\end{array} \\
\hline\left(q^{2}+1\right)(q+1)^{2} & \chi_{3} & 1 & 2^{2 n}
\end{array} \\
& b_{4}\left(\frac{\left(m^{\prime}-1\right)\left(m^{\prime}-3\right)}{8} \text { blocks }\right): \\
& \begin{array}{l|c|c|c}
\text { degrees } & \text { chars. } & & \begin{array}{c}
\text { no. of } \\
\text { chars. }
\end{array} \\
\hline\left(q^{2}+1\right)(q-1)^{2} & \chi_{4} & 1 & 2^{2 n^{\prime}}
\end{array} \\
& b_{5}\left(\frac{(m-1)\left(m^{\prime}-1\right)}{4} \text { blocks }\right): \\
& \begin{array}{c|c|c|c}
\text { degrees } & \text { chars. } & & \begin{array}{c}
\text { no. of } \\
\text { chars. }
\end{array} \\
\hline q^{4}-1 & \chi_{5} & 1 & 2^{d}
\end{array}
\end{aligned}
$$

TABLE 1. Blocks with abelian defect groups. 


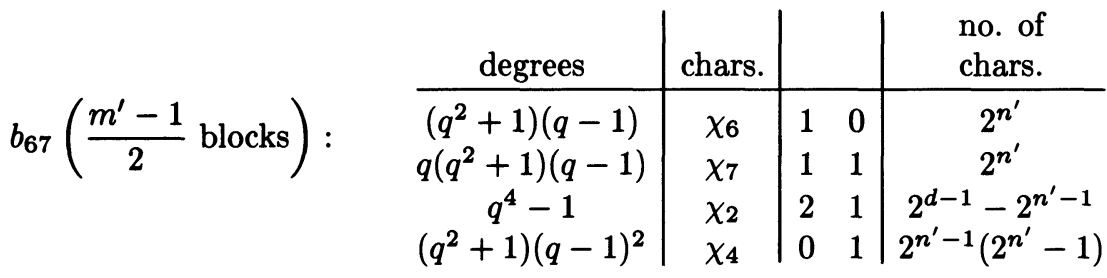

$$
\begin{aligned}
& b_{89}\left(\frac{m-1}{2} \text { blocks }\right):
\end{aligned}
$$

\begin{tabular}{c|c|cc|c} 
degrees & chars. & & & $\begin{array}{c}\text { no. of } \\
\text { chars. }\end{array}$ \\
\hline$\left(q^{2}+1\right)(q+1)$ & $\chi_{8}$ & 1 & 0 & $2^{n}$ \\
$q\left(q^{2}+1\right)(q+1)$ & $\chi_{9}$ & 1 & 1 & $2^{n}$ \\
$q^{4}-1$ & $\chi_{2}$ & 0 & 1 & $2^{d-1}-2^{n-1}$ \\
$\left(q^{2}+1\right)(q+1)^{2}$ & $\chi_{3}$ & 2 & 1 & $2^{n-1}\left(2^{n}-1\right)$
\end{tabular}

TABLE 2. Blocks of types $b_{67}, b_{89}$.

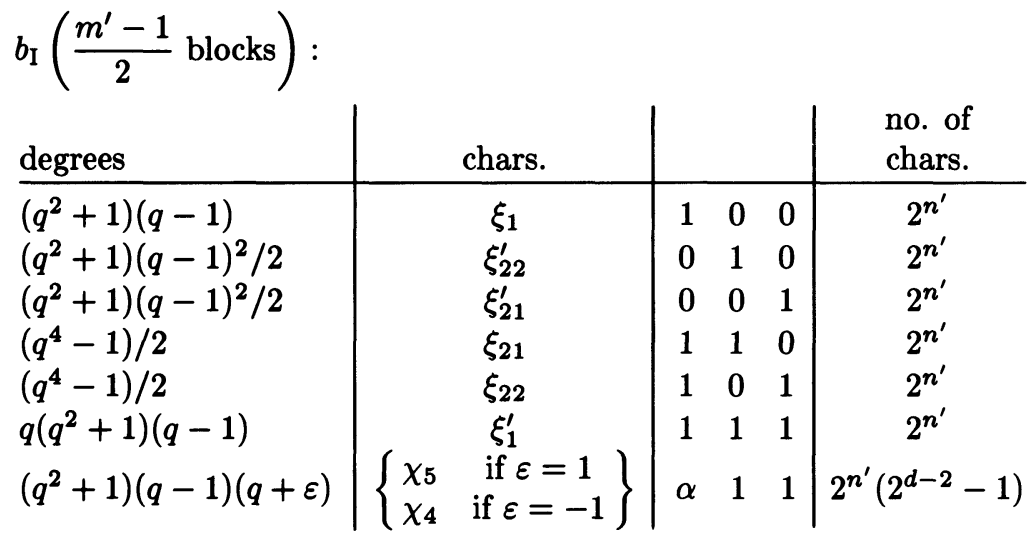

$b_{\mathrm{III}}\left(\frac{(m-1)}{2}\right.$ blocks $):$

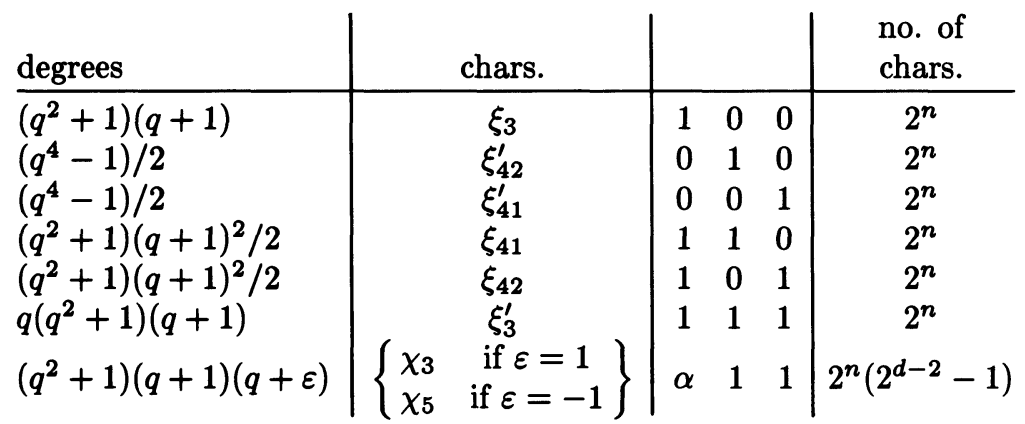

TABLE 3 . Blocks of types $b_{\mathrm{I}}, b_{\mathrm{III}}$. 


\begin{tabular}{c|c|ccccccc|c} 
degrees & & & & & & & & no. of \\
chars.
\end{tabular}

TABLE 4. The principal block $b_{0}$.

\section{REFERENCES}

1. W. Feit, The representation theory of finite groups, North-Holland, Amsterdam, 1982.

2. G. Hiss, On the decomposition numbers of $G_{2}(q)$, Preprint. 1986.

3. J. Shamash, Blocks and Brauer trees for groups of type $G_{2}(q)$, Ph.D. Thesis, Yale Univ.,

4. B. Srinivasan, The characters of the finite symplectic group $\operatorname{Sp}(4, q)$, Trans. Amer. Math. Soc. 131 (1968), 488-525.

5. D. L. White, The 2-blocks and decomposition numbers of $\mathrm{Sp}(4, q), q$ odd, $\mathrm{Ph} . \mathrm{D}$. Thesis, Yale Univ., 1987.

Department of Mathematics, Yale University, New haven, ConnectiCUT 06520

Current address: Department of Mathematics, University of Illinois at Chicago, Chicago, Illinois 60680 